\title{
MÉTODOS DE ESTIMACIÓN NO LINEAL APLICADOS AL PROBLEMA DE EXPECTATIVAS DE INFLACIÓN
}

\section{NONLINEAR ESTIMATION METHODS APPLIED TO A PROBLEM OF EXPECTED INFLATION}

\author{
Mauricio Arriagada-Benítez ${ }^{*} \quad$ Héctor Valdés-González ${ }^{2} \quad$ Liliana Pedraja-Rejas $^{1}$ \\ Recibido 20 de febrero de 2008, aceptado 22 de julio de 2009 \\ Received: February 20, 2008 Accepted: July 22, 2009
}

\begin{abstract}
RESUMEN
En este trabajo se describe y analiza, luego de tener la ecuación que relaciona la dinámica de las expectativas de la tasa de interés real y de la inflación, el filtro extendido de Kalman. Asimismo, se realiza la estimación de la inflación ex ante para una serie de datos preestablecida. Se efectúa una comparación con el método de estimación de horizonte móvil utilizado en situaciones cuando producto de las incertidumbres paramétricas del modelo éste se torna no lineal. La aplicación de estos métodos a datos reales permite concluir que las estimaciones efectuadas a través del método de horizonte móvil, combinado a un algoritmo heurístico de optimización logran los mejores resultados.
\end{abstract}

Palabras clave: Filtrado de Kalman, método MHSE, inflación, modelos econométricos.

\section{ABSTRACT}

This work describes and analyses the Extended Kalman Filter with regard to an equation that relates the dynamic of the expected real interest rates and inflation. An estimation of the ex ante inflation for a preestablished data set is carried out. This is compared with the same calculation using a moving horizon estimation method for situations that are non lineal due to parametric uncertainties of the model. From the application of these methods to real data, it can be concluded that the estimations based on the moving horizon method, combined with a heuristic optimization algorithm, yield better results.

Keywords: Kalman Filter, MHSE method, inflation, econometric models.

\section{INTRODUCCIÓN}

Este artículo se centra en la aplicación de técnicas de estimación de estados como el EKF (Extended Kalman Filter, por su sigla en inglés) y el método MHSE (Moving Horizon State Estimation por su sigla en inglés) a problemas econométricos. Problema de estimación que pretende obtener las expectativas de inflación para un período determinado de años a partir de la información contenida en la dinámica conjunta de la tasa de interés nominal y de la inflación observada [1, 3-4, 20].

Todo modelamiento exige la consideración de supuestos. Así, para poder obtener expectativas de inflación se debe considerar: (i) la existencia de mercados eficientes [9], (ii) la racionalidad en la conformación de las expectativas de inflación, lo cual supone errores de pronóstico insesgados y no autocorrelacionados [16], y (iii) la influencia de la inflación esperada en la evolución de la tasa de interés nominal.

Este último supuesto, se fundamenta en el resultado del modelo Mundell-Tobin donde se observa una relación negativa entre la inflación esperada y la tasa de interés real ex ante [7]. Lo cual indica, que tomando como punto de partida la teoría clásica de la demanda por dinero, una recomposición de portafolio de dinero por activos financieros que rinden una tasa de interés, que incluye una compensación por inflación esperada, genera una reducción en la tasa de interés real esperada $[8,20]$.

1 Escuela Universitaria de Ingeniería Industrial, Informática y de Sistemas. Universidad de Tarapacá. Av. 18 de Septiembre 2222, Campus Saucache, Arica, Chile. E-mail: lpedraja@uta.cl (* Alumno del programa de Magíster en Ingeniería de Software).

2 Universidad Andrés Bello. Facultad de Ingeniería. Escuela de Industrias, Ingeniería en Automatización y Robótica. Av. República 239. Santiago, Chile. E-mail: hvaldes@unab.cl 
En [20], a diferencia de [15], no se considera el supuesto de que las tasas de interés reales esperada y observada presenten la misma dinámica en el largo plazo debido a que el procedimiento econométrico utilizado permite la consideración de variables no observadas, y por tanto el problema se aborda utilizando la técnica clásica de filtrado de Kalman. En este trabajo, a su vez, se considera la variación de los parámetros estructurales que gobiernan la dinámica de la tasa de interés real ex ante y de la inflación esperada a partir de la tasa de interés nominal y de la inflación observada, pero siempre trabajando con un modelo espacio estado lineal del proceso.

Otra publicación [5] aplica la técnica de filtrado de Kalman para observar la relación entre tipos de interés real, inflación y estructura de término del tipo de interés, bajo hipótesis de expectativas. Por su parte, se sabe que la inflación consiste en el crecimiento generalizado y continuo de los precios de los bienes, servicios y factores productivos de un país. Por lo tanto implica la reducción de la capacidad adquisitiva del dinero; diversos son los autores que argumentan que, en su mayoría, los más importantes costos de la inflación están ligados a su imperfecta predictibilidad [10]. En efecto, uno de los factores a través del cual un mayor nivel de inflación podría afectar el nivel de actividad económica es la incertidumbre sobre los niveles futuros de la inflación. Esta volatilidad de la inflación, además de obstaculizar el cálculo económico, aplica importantes costos sociales en la economía [19]. Se debe notar que en este caso, también se opta por trabajar con modelos lineales, desechando las no linealidades propias del proceso.

En este contexto, se observan diversos trabajos que han orientado el análisis de la estructura de las tasas de interés hacia la utilización de modelos probabilísticos. Así, [21, 24] valida el hecho de utilizar modelos espacio estado para proyectar la inflación por sus evidentes ventajas. Asimismo, se han desarrollado estudios para estimar la tasa de inflación influenciada por el tipo de interés nominal, bajo el análisis de la no linealidad [12], desarrollándose mejoras en la estimación usando procedimientos para errores no-normales [22]. Sin embargo, subsiste el problema de que el EKF no es robusto frente a perturbaciones externas y que la convergencia de sus estimaciones no puede ser asegurada.

El presente artículo, a diferencia de trabajos previos [15, 20] y [4], considera precisamente la no linealidad del proceso de expectativas de inflación. Con este propósito, y para considerar la naturaleza no lineal del proceso, se suponen ruidos estocásticos propios de un proceso econométrico [13, 17], para así abordar el problema a través del EKF (que opera con un modelo linealizado y en torno a un punto de operación) y MHSE (que trabaja con modelos no lineales directamente y cuya convergencia depende de la convergencia del método de optimización utilizado), comparando estos resultados entre sí, y obteniendo con ello resultados cuantificados favorables en la estimación de la inflación real ex ante con el método MHSE.

\section{MODELO ECONOMÉTRICO DE EXPECTATIVAS DE INFLACIÓN}

Como se indica en $[17,20]$, el modelo estructural, conformado por las representaciones dinámicas de la tasa de interés real ex ante y de la inflación esperada, parte de la ecuación de Fisher [11], donde se puede identificar que la tasa de interés en un determinado período es función de las expectativas de los retornos reales y de la inflación.

Siguiendo la investigación propuesta por ejemplo en $[15,4,16]$, se observa que es posible representar la dinámica de las expectativas de la tasa de interés real y de la inflación a partir de las ecuaciones constitutivas descritas por (1) y (2), las cuales constituyen el punto de partida de la representación del modelo. Considerando que: $z(L)=z_{1} L^{1}+\cdots+z_{p} L^{p}$ para cualquier variable $z$.

$$
\begin{gathered}
r_{t}=k_{1}+\Phi(L) r_{t}+\psi_{0} \pi_{t}^{e}+\Psi(L) \pi_{t}^{e}+\xi(L) r_{t}+\varepsilon_{1 t} \\
\pi_{t}^{e}=k_{2}+\alpha(L) r_{t}+\beta(L) \pi_{t}^{e}+\gamma(L) \pi_{t}+\varepsilon_{2 t}
\end{gathered}
$$

Se suponen además, considerando (1) y (2), las siguientes propiedades sobre los términos de perturbación, a saber:

$$
\begin{aligned}
& \left.\left.\mathrm{E}\left[\varepsilon_{1 t}\right]=\mathrm{E} \mid \varepsilon_{1 t} r_{t-j}\right\rfloor=\mathrm{E} \mid \varepsilon_{1 t} \pi_{t+1-j}^{e}\right\rfloor=\mathrm{E}\left[\varepsilon_{1 t} \pi_{t-j} \mid=0\right. \\
& \forall j \geq 1 \\
& \left.\mathrm{E}\left[\varepsilon_{2 t}\right]=\mathrm{E}\left[\varepsilon_{2 t} r_{t-j}\right]=\mathrm{E} \mid \varepsilon_{2 t} \pi_{t-j}^{e}\right\rfloor=\mathrm{E}\left[\varepsilon_{2 t} \pi_{t-j}\right]=0 \\
& \forall j \geq 1
\end{aligned}
$$

Donde (3) y (4) garantizan una estructura dinámica conjunta estable. Además, como se plantea en [15], 1985, es posible escribir: 
$\mathrm{E}\left\lfloor\varepsilon_{i, t} \varepsilon_{i, t-j}\right\rfloor=\mathrm{E}\left\lfloor\varepsilon_{i, t} \varepsilon_{k, t-j+1}\right\rfloor=\mathrm{E}\left\lfloor\varepsilon_{i, t} e_{t-j}\right\rfloor=0$

$\forall i . k=1,2 \quad \forall j \geq 1$

Donde $\pi_{\mathrm{t}}$ : Inflación anual observada en el período $(\mathrm{t})$; $\pi_{t}^{e}$ : Expectativa de inflación en el período ( $\mathrm{t}$ ) basadas en la información hasta período $(\mathrm{t}) ; e_{t}=\pi_{t}-\pi_{t}^{e}$ error de pronóstico en el mercado en el período $(\mathrm{t}) ; i_{t}$ : Tasa de interés nominal de los certificados de depósito a 90 días en el período $(\mathrm{t}) ; r_{t}=i_{t}-\pi_{t}^{e}$, tasa de interés real ex ante en el período $(\mathrm{t})$.

En la ecuación (1), la tasa de interés real ex ante, $r_{t}$, es función de su historia $\phi(L) r_{t}$ de la inflación esperada contemporánea $\psi_{0} \pi_{t}^{e}$ y rezagada $\psi(L) \pi_{t}^{e}$, como también, de la evolución pasada de la inflación observada $\xi(L) \pi_{t}$ y de un término de perturbación aleatorio. La inflación esperada, $\pi_{t}^{e}$ depende de las evoluciones pasadas de la tasa de interés real ex ante $\alpha(L) r_{t}$, la inflación esperada $\beta(L) \pi_{t}^{e}$ y la observada $\gamma(L) \pi_{t}$ y de un término aleatorio de error. Así como señala [15], adicional a los supuestos que llevan a la estabilidad estructural dinámica conjunta de las expectativas de inflación y la tasa de interés real ex ante, ha de considerarse un mercado financiero eficiente en cuanto a que el conjunto de información de los agentes en el período (t) incluye como mínimo rezagos de la tasa de interés nominal, de la inflación esperada y de la inflación observada. Es decir, la inclusión de la tasa de interés real ex ante, contemporánea y rezagada, en el conjunto de información relevante para la conformación de las expectativas por parte de los agentes económicos.

Finalmente, y de acuerdo a la definición de la tasa de interés real ex ante, ahora la ecuación (1) puede ser reescrita en términos de la inflación esperada y de la tasa de interés nominal, teniendo así:

$i_{t}-\pi_{t}^{e}=k_{1}+\Phi(L)\left(i_{t}-\pi_{t}^{e}\right)+\psi_{0} \pi_{t}^{e}+\Psi(L) \pi_{t}^{e}+\xi(L) r_{t}+\varepsilon_{1 t}$

Donde al despejar la tasa de interés nominal se obtiene:

$i_{t}=k_{1}+\left(1+\psi_{0}\right) \pi_{t}^{e}+(\Psi(L)-\Phi(L)) \pi_{t}^{e}+\Phi(L) i_{t}+\xi(L) r_{t}+\varepsilon_{1 t}(7)$

Con el sistema de ecuaciones conseguido desde (7) y (2) y la definición del error de pronóstico de mercado, ahora puede ser presentado el modelo en términos de una representación del tipo espacio-estado [15, 4].

$$
\begin{aligned}
& x_{t+1}=f(x)+\tilde{w}_{t+1} \\
& y_{t}=g(x)+\tilde{v}_{t}
\end{aligned}
$$

Donde $f($.) y $g($.) son funciones no lineales, además el vector de estados queda determinado por $x_{t}=\left(\pi^{e}, \pi^{e}, \cdots, \pi^{e}\right)^{T}$ y la salida $y_{t}($.$) del sistema (8) queda a su vez determinada$ por: $y_{t}=\left(i_{t}, \pi_{t}\right)^{T}$ que son variables dependientes. $\tilde{w}_{t+1} \mathrm{y}$ $\tilde{v}_{t+1}$ representan términos de perturbación estocástica.

Los parámetros del modelo (2) y (7), representados por (8), deben ser estimados y para ello se utiliza el ejemplo y los datos descritos en $[15,4,20]$.

\section{FILTRO EXTENDIDO DE KALMAN}

El problema de filtrado de Kalman se define como un procedimiento recursivo que permite calcular el estimador óptimo del vector de estado en cada momento del tiempo con base en la información disponible en el momento $t-1$, y actualizar con la información adicional disponible en el momento t, dichas estimaciones. En otros términos, dicho procedimiento permite encontrar la mejor estimación del vector de estados que considera la expectativa de inflación como variable de estado. La forma recursiva del filtro extendido de Kalman puede ser expresada a través del siguiente sistema de ecuaciones, donde todos los símbolos en la formulación denotan matrices o vectores $[3,14,23,24]$.

a) Predicción del estado [3, 2, 18]:

$$
x_{n+1 / n}=\Phi\left(n+1, n, x_{n / n-1}, u_{n}\right)
$$

Donde:

$$
\begin{aligned}
\Phi(n+1, & \left.n, x_{n / n-1}, u_{n}\right)= \\
& A_{n}\left(x_{n / n}\right) x_{n / n}+B_{n}\left(x_{n / n}\right) u_{n}
\end{aligned}
$$

b) Estimación del error en la matriz de covarianza:

$$
\begin{aligned}
P_{n+1 / n}= & \left.\left.\frac{\partial \Phi}{\partial x}\right|_{x=x_{n / n}} \cdot P_{n / n} \cdot \frac{\partial \Phi^{T}}{\partial x}\right|_{x=x_{n / n}} \\
& +\Gamma_{n} Q \Gamma_{n}^{T}
\end{aligned}
$$


Donde:

$$
\Gamma=\int_{n}^{n+1} \Phi\left(t_{n+1}, \tau\right) G(\tau) d \tau
$$

y el valor inicial de $P_{n / n}$ es una matriz de valores constantes.

c) Cálculo de la ganancia de Kalman

$$
\begin{gathered}
K_{n}=\left.P_{n / n-1} \frac{\partial H^{T}}{\partial x}\right|_{x=x_{n / n-1}} \cdot \\
\left(\left.\left.\frac{\partial H}{\partial x}\right|_{x=x_{n / n-1}} \cdot P_{n / n-1} \frac{\partial H^{T}}{\partial x}\right|_{x=x_{n / n-1}}\right)
\end{gathered}
$$

Donde:

$$
H\left(x_{n / n-1}, n\right)=C_{n}\left(x_{n / n-1}\right) x_{n / n-1}
$$

d) Estimación del estado

$$
\hat{x}_{n / n}=x_{n / n-1}+K_{n}\left(y_{n}-H\left(x_{n / n-1}, n\right)\right)
$$

e) Actualización del error de la matriz de covarianza

$$
P_{n / n}=P_{n / n-1}-\left.K_{n} \cdot \frac{\partial H}{\partial x}\right|_{x=x_{n / n-1}} P_{n / n-1}
$$

\section{MÉTODO DE HORIZONTE MÓVIL}

El método MHSE consiste en reemplazar un problema dinámico de estimación por un problema estático de optimización no lineal. Problema cuyo criterio es definido como la suma del error cuadrático entre las medidas de la salida y las salidas estimadas por el modelo del proceso sobre un horizonte de tiempo predefinido. La minimización de este criterio apunta a determinar el estado inicial $\hat{x}(t)$, al inicio del horizonte, tal que este conduzca a una minimización entre lo observado y la trayectoria predicha sobre el horizonte de tiempo. Se debe notar que en el caso lineal, el problema es fácil de resolver y una solución formal puede ser establecida. Sin embargo, no linealmente, una solución analítica no puede ser establecida para el problema y la resolución del problema debe ser obtenida numéricamente a través de optimización no lineal, que es la base del método MHSE.

Primero, se va a describir el criterio de optimización de MHSE que debe ser minimizado. Se considerará el modelo no lineal descrito en (8), con una única salida para simplificar la notación, donde $Y_{k}=\left[\begin{array}{llll}y_{s h} & y_{s h+1} & \cdots & y_{k}\end{array}\right]^{T}$ son las medidas de la salida sobre el horizonte considerado, con $y_{i}$ equivalente a decir $y\left(t_{i}\right)$. Los subíndices sh y $k$ representan el origen del horizonte y el tiempo actual respectivamente (al final del horizonte de largo $l h, k=s h+l h$ ). Se denota como $\hat{x}_{s h}$ al vector de estados al inicio del horizonte de tiempo, tal que a partir de este estado es predicha la trayectoria $Y_{k}$ sobre el horizonte de tiempo predefinido.

$$
\left[\begin{array}{llll}
\hat{y}_{s h} & \hat{y}_{s h+1} & \ldots & \hat{y}_{k}
\end{array}\right]^{T}=G\left(\hat{x}_{s h}, t_{s h}, t_{k}\right)
$$

Donde $G\left(\hat{x}_{s h}, t_{s h}, t_{k}\right)$ se define como:

$$
G\left(x, t_{i}, t_{j}\right)=\left[\begin{array}{c}
g\left(x\left(t_{i}\right)\right) \\
g\left(F\left(x, t_{i}, t_{i+1}\right)\right) \\
\vdots \\
g\left(F\left(x, t_{i}, t_{j}\right)\right)
\end{array}\right]
$$

Donde $F\left(x, t_{i}, t_{l}\right)$ queda a su vez expresado por:

$$
F\left(x, t_{i}, t_{l}\right)=x\left(t_{i}\right)+\int_{t_{i}}^{t_{l}} f(x(\tau), u(\tau)) d \tau
$$

El principio del método es entonces, determinar el vector de estados $\hat{x}_{s h}$, tal que $\hat{x}_{s h}^{*}$ cumpla con:

$$
\hat{x}_{s h}^{*}=\arg \min \left[J\left(\hat{x}_{s h}\right)\right]
$$

El criterio $J($.$) descrito puede reescribirse como$ $J\left(\hat{x}_{s h}\right)=\frac{1}{2} R_{k}^{T} R_{k}$. Donde $R_{k}$ es el vector de residuos, a su vez expresado como $R_{k}=Y_{k}-G\left(\hat{x}_{s h}, t_{s h}, t_{k}\right)$. Notar que la observabilidad del sistema (8) queda restringida a la existencia y unicidad de la solución del problema (19). 


\section{Algoritmo MHSE básico}

1) Inicialización del vector de estados $\hat{x}_{s h}$.

2) Búsqueda del vector de estados óptimo $\hat{x}_{s h}^{*}$.

3) Cálculo del vector de estados actual.

$$
\hat{x}_{k}^{*}=F\left(\hat{x}_{s h}^{*}, t_{s h}, t_{k}\right)
$$

4) Volver al paso 2) para calcular $\hat{x}_{k+1}^{*}$, con

$$
s h \leftarrow s h+1
$$

$$
\hat{x}_{s h}=F\left(\hat{x}_{s h}^{*}, t_{s h}, t_{s h+1}\right) \text {. }
$$

La minimización del criterio (19) es desarrollada a través del método heurístico Simulated Annealing (SA) [6]. La convergencia de las estimaciones realizadas con MHSE está directamente relacionada con la convergencia de la optimización efectuada en (19). Para detalles ver [1].

\section{RESULTADOS Y DISCUSIÓN}

Utilizando los valores de los parámetros establecidos en [15] para simular el modelo representado por (2) y (7), y los datos publicados en [20], es posible obtener estimaciones para las "expectativas de inflación" o $\pi_{t}^{e}$.

La Figura 1, muestra una comparación entre la inflación real medida (línea azul), las expectativas de inflación estimada a través de un EKF (línea en negro) y en color rojo la correspondiente a la estimación efectuada con el método MHSE. Es posible notar que, dada la utilización de un horizonte de tiempo largo para la estimación $(l h=20$ trimestres), se puede observar que las estimaciones efectuadas a través del método MHSE casi se superponen por completo a la medida real dando cuenta de la correcta estimación y ventaja de la estimación no lineal por sobre la que se realiza con un modelo linealizado, como lo es el caso del EKF.

La Figura 2, considera las mismas estimaciones, pero bajo una perturbación estocástica $\tilde{v}_{t+1}($ en (8)) de un 5\% de ruido gaussiano (distribución Normal $(0,1)$ ), sobre la tasa de interés nominal de los certificados de depósito a 90 días, y la inflación real observada en el período $(l h=3$ trimestres).

Entre las implicancias que traen consigo estos resultados, y comprando ventajas y desventajas principales se encuentra que:

a) Se aprecia que con el EKF se obtienen buenos resultados de estimación, sin embargo estos resultados pertenecen a un caso ideal, es decir, sin errores ni perturbaciones y con un modelo linealizado, que es válido solo en torno a un punto de operación.

b) Por otra parte, a través del método de MHSE, acoplado con el algoritmo heurístico de optimización no lineal, denominado Simulated Annealing, también se obtienen buenos resultados de convergencia. Sin embargo, en este caso se trabaja con un modelo no lineal y por tanto más realista del proceso econométrico involucrado. De hecho, los resultados obtenidos muestran claramente que MHSE presenta ventajas comparativas, en particular debido a la existencia de una prueba matemática de su convergencia [1]. 


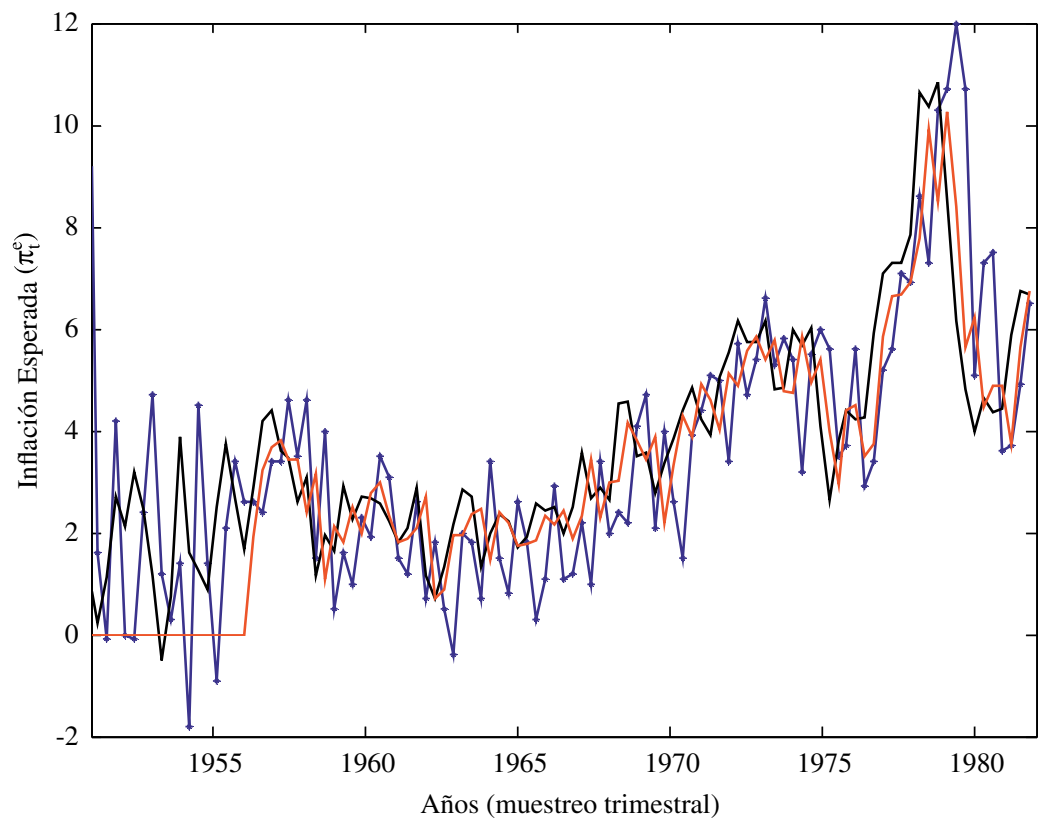

Figura. 1. Comparación entre la inflación real medida (línea azul), las expectativas de inflación estimada a través de un EKF (línea en negro) y en color rojo la correspondiente a la estimación efectuada con el método MHSE.

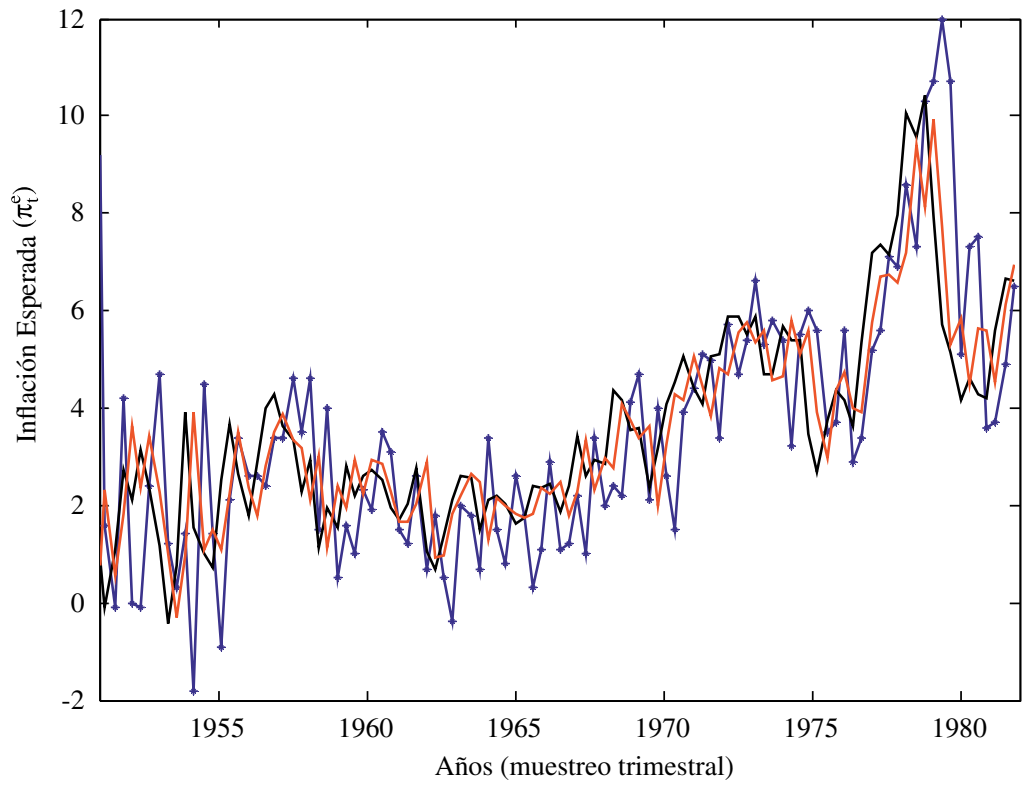

Figura. 2. Estimaciones como en Figura 1, bajo una perturbación estocástica $v_{t+1}$ de un $5 \%$ de ruido gaussiano, sobre la tasa de interés nominal de los certificados de depósito a 90 días, y la inflación real observada en el período. 


\section{CONCLUSIONES}

En este trabajo se presenta una estrategia basada en un algoritmo de estimación no lineal, para efectuar estimación de la variable inflación en el tiempo, a través de mediciones indirectas. Se analizan y dejan por sentadas las bases necesarias para realizar estimación no lineal a través de la técnica MHSE, siendo uno de sus aspectos principales la optimización no lineal asociada. Algoritmo que permite relacionar con mejor precisión la ecuación que conecta la dinámica de las expectativas de inflación con la tasa de interés real. En particular, este trabajo presenta su uso con un modelo no lineal y por tanto más realista del proceso econométrico involucrado. De hecho, los resultados obtenidos muestran claramente que MHSE presenta ventajas comparativas, en particular debido a la existencia de una prueba matemática de su convergencia y a la no dependencia de un punto de operación, como lo es el caso EKF.

Comparaciones con el EKF han sido efectuadas, para realizar la estimación de la inflación ex ante, logrando mejores resultados con la técnica no lineal MHSE. Los resultados en simulación indican la gran aplicabilidad de técnica expuesta, haciendo notar que la proposición considera la no linealidad intrínseca del problema y no la de un modelo linealizado, con las ventajas en precisión que ello conlleva.

\section{REFERENCIAS}

[1] L. Boillereaux and J.M. Flaus. "A new approach for designing model-based indirect sensors". IEEE Transactions on control systems technology. Vol. 8 No 4, pp. 601-608. July 2000.

[2] E. Brookner. "Tracking and Kalman Filtering Made Easy". John Wiley \& Sons. Estados Unidos. 1998.

[3] R.G. Brown and P.Y.C. Hwang. "Introduction to Random Signal and Applied Kalman Filtering: with MATLAB Exercises and Solutions". John Wiley \& Sons. Tercera edición. Estados Unidos. 1997.

[4] E. Burmeister, K.D. Wall and J.D. Hamilton. "Estimation of Unobserved Expected Monthly Inflation Using Kalman Filtering". Journal of Business and Economic Statistics. Vol. $4 \mathrm{~N}^{\circ} 2$, pp. 147-160. April 1986.
[5] L.H. Chen. "Inflation and Real Short-Term Interest Rates-A Kalman Filter Analysis of the Term Structure". Applied Economics. Vol. 33 № 7, pp. 855-861. June 2001.

[6] A. Díaz, F. Glover, H.M. Ghaziri, J.L. González, M. Laguna, P. Moscato, y F.T. Tsen. "Optimización Heurística y Redes Neuronales". Editorial Paraninfo. Madrid, España. Julio 1996.

[7] R. Dornbusch y S. Fischer. "Macroeconomía". McGraw-Hill. Estados Unidos. 2002.

[8] E.F. Fama and M.R. Gibbons. "Inflation, real returns and capital investment". Journal of Monetary Economics. Vol. 9 No 3, pp. 297-323. 1982.

[9] E.F. Fama and M.R. Gibbons. "The Information in Long-Maturity Forward Rates". American Economic Review. Vol. 77 No 3, pp. 680-692. September 1987.

[10] C. Fernández. "Inflation and Welfare: An Application to Chile". Cuadernos de Economía. Vol. 36 N 107, pp. 519-544. 1999.

[11] I. Fisher. "The Theory of Interest". MacMillan. Primera edición. New York, Estados Unidos. 1930.

[12] J.A. Frankel. "A Technique for Extracting a Measure of Expected Inflation from the Interest Rate Term Structure". The Review of Economics and Statistics. Vol. $64 \mathrm{~N}^{\circ}$ 1, pp. 135-142. February 1982.

[13] V. Gómez. "Three Equivalent Methods for Filtering Finite Nonstationary Time Series". Journal of Business and Economic Statistics. Vol. $17 \mathrm{~N}^{\mathrm{o}} 1$, pp. 109-116. January 1999.

[14] M. Grewal and A. Andrews. "Kalman Filtering: Theory and Practice". Prentice Hall. 1993.

[15] J.D. Hamilton. "Uncovering Financial Market Expectations of Inflation". Journal of Political Economy. Vol. 93 N 6, pp. 1224-1241. December 1985.

[16] J.D. Hamilton. "State-Space Models". Handbook of Econometrics. Vol. 4 No 1, pp. 3039-3080. 1986. 
[17] J.D. Hamilton. “Time Series Analysis". Princeton University Press. Estados Unidos. 1994.

[18] Y.R. Kim, S.K. Sul and M.H. Park. "Speed sensorless vector control of induction motor using extended Kalman filter". Sensorless Control of AC Motor Drives. New York: IEEE Press, pp. 215-223. 2002.

[19] I. Magendzo. "Inflación e Incertidumbre Inflacionaria en Chile". Documentos de trabajo del Banco Central de Chile. No 15. Octubre 1997.

[20] M. Misas y D.M. Vásquez. "Expectativas de Inflación en Colombia: Un Ejercicio Econométrico". Banco de la República, Subgerencia de Estudios Económicos. No 212. Colombia. 2002.
[21] F. Nadal de Simona. "Proyección de la inflación en Chile". Economía Chilena. Vol. 4 No 3, pp. 59-85. Diciembre 2001.

[22] P. Silvapulle and R. Hewarathna. "Robust estimation and inflation forecasting". Applied Economics. Vol. $34 \mathrm{~N}^{\circ}$ 18, pp. 2277-2282. Diciembre 2002.

[23] M.J. Valderrama y J.C. Ruiz. "Filtrado de Kalman: Aplicaciones en Economía e Ingeniería”. Editorial Universidad de Barcelona. Barcelona, España. 1996.

[24] K. A. Holmes and R. Faff. "Estimating the performance attributes of Australian multi-sector managed funds within a dynamic Kalman filter framework". International Review of Financial Analysis. Vol. 17 No 5, pp. 998-1011. 2008. 